\title{
Investigation on In-band Interference Effect and Out-of- band Interference Mechanism of B1I Navigation Receiver
}

\author{
Qinglong ZHANG, Yuming WANG, Erwei CHENG, Liyun MA, Yazhou CHEN \\ National Key Laboratory on Electromagnetic Environment Effects, \\ University of Shijiazhuang Campus of Army Engineering University, Heping West Road 97, Shijiazhuang, China \\ zq12928@126.com,ymking2006@163.com,ew_cheng@163.com,271425024@qq.com,chen_yazhou@sina.com
}

Submitted December 28, 2020 / Accepted June 10, 2021

\begin{abstract}
Aiming at the problem that the Beidou B1I navigation receiver is susceptible to continuous-wave interference (CWI), it is of great significance to investigate the interference effect and mechanism of the BII navigation receiver. With the threshold of carrier-to-noise ratio $\left(C / N_{0}\right)$ as the criterion of satellite tracking missing, the CWI injection experiment is conducted on a kind of BII navigation receiver. The results indicate that the power spectrum of the satellite signal affects the CWI's ability to interfere with each satellite. Then, a prediction model of satellite tracking loss under dual-frequency CWI is proposed, and the accuracy of the model is verified through experiments. The experiment results demonstrate that the power combination between the dual-frequency CWI is negatively correlated, and the error of this model is less than $2 \mathrm{~dB}$. Finally, the sensitive frequency bands of the navigation receiver under out-of-band CWI are obtained through experiments. It is confirmed by theoretical and simulation analysis that the mixer is the main non-linear circuit generating these sensitive frequency bands. Furthermore, improving the selectivity of the radio frequency $(R F)$ front-end circuit can enhance the performance of anti out-of-band interference.
\end{abstract}

\section{Keywords}

Beidou B1I navigation receiver, CWI, dualfrequency, sensitive frequency bands, mixer

\section{Introduction}

Nowadays, satellite navigation technology has penetrated all aspects of life [1]. However, the satellite navigation signal on the surface of the earth is extremely weak. The lower in-band interference signal power will reduce the satellite's $C / \mathrm{N}_{0}$, deteriorating the receiver's positioning performance. Studying the influence of CWI [2] on the navigation receiver is conducive to the development of interference technology and anti-interference technology.

The positioning result of the navigation receiver is a comprehensive performance of all the tracking loops inside it. Any of the satellite tracking missing may affect the positioning result. The interference not only reduces the amplitude of the satellite signal by influencing the gain circuits in the navigation receiver [3] but also weakens the tracking performance on satellite signals in the process of signal correlation processing. At present, the investigation on the performance of satellite navigation receivers under electromagnetic interference (EMI) is divided into two aspects [4]: 1) the theoretical analysis on the effect of the tracking performance under the EMI, and 2) the electromagnetic compatibility (EMC) of the navigation receiver.

There are many studies on the effect of the tracking performance under the EMI. The calculation formula of the $\mathrm{C} / \mathrm{N}_{0}$ was derived under $\mathrm{CWI}$ and pulse interference [5]. The analytical formula of the maximum tracking error under single frequency interference was deduced, and the influence of code tracking error under different modulation mode and interference conditions were analyzed [6]. The analytical expressions of various performance parameters under EMI are provided [7], [8], such as the effective $\mathrm{C} / \mathrm{N}_{0}$, code tracking (DLL) error, and carrier phase tracking (PLL) error. Besides, the effect of the DLL error under CWI and multipath interference were analyzed [9]. The code tracking error of the navigation receiver under the CWI was explored under the consideration of more external factors, such as interference-to-signal ratio (ISR) and RF front-end bandwidth [10]. However, the electromagnetic environment is becoming more complex, and navigation receivers will face dual-frequency interference signals. In this case, the navigation receiver may have been interfered within the known safety margin of single-frequency interference while satellite tracking has been missed. Therefore, it is necessary to conduct dual-frequency EMI modeling and effect research.

The research on EMC mainly focuses on receiver positioning performance while neglecting the mechanism of positioning failure. Researchers conducted CWI and ultra-wideband radiation experiments for three different types of GPS receivers and compared the interference threshold and damage threshold of different receivers [11]. Liu et al. proposed the sensitivity experiment method and uncertainty evaluation method for the Beidou navigation 
receiver [12], [13]. Moreover, the components that may be affected by out-of-band interference have been handled [14], but it does not present a specific case analysis.

The investigation in this paper mainly emphasizes the navigation receiver of the B1I signal. The main contributions are described as follows.

1) In Sec. 2, the power spectrum density (PSD) of B1I signals is analyzed, and the electromagnetic susceptibility (EMS) criterion of the satellite tracking missing is introduced. According to the experimental results, the interference thresholds of different satellites are revealed.

2) In Sec. 3, the prediction model is established for the satellite tracking missing under the dual-frequency CWI. Besides, the model was verified by the dual-frequency CWI injection experiment.

3) In Sec. 4, the experiment of out-of-band interference on the B1I receiver is performed, and the sensitive frequency bands are obtained. The mechanism that out-ofband interference causes the navigation receiver positioning failure is explored through experiment and simulation.

\section{Analysis on the PSD of B1I Signal and the EMS Criterion}

\subsection{Analysis on the Ranging Code of B1I Signal}

The ranging code of the Beidou B1I signal is a gold code generated by two linear sequences G1 and G2 [15]. The modulation method is quadrature phase-shift keying (QPSK), which contains I branch and Q branch. However, China has only published the signal structure of B1I at present, and the B1Q branch is not available. In this case, the QPSK signal is binary phase-shift keying (BPSK) modulation [4]. Considering the influence of signal modulation, the equation of the PSD of the ranging code is

$$
\mathrm{G}_{\mathrm{PRN}}(f)=\frac{1}{N^{2}} \sum_{n=-\infty}^{\infty}\left|C_{\mathrm{PRN}}(n)\right|^{2} \operatorname{sinc}^{2}\left(\frac{n}{N}\right) \delta(f-n \cdot \Delta f)
$$

where $N$ represents the code length of ranging code, $C_{\text {PRN }}(n)$ denotes the $N$-point Fourier transform coefficients, $\Delta f$ indicates the range code repetition frequency.

Beidou navigation system (BDS) has three orbiting satellites: geostationary earth orbit (GEO), geosynchronous satellite orbit (IGSO), and medium earth orbit (MEO). The PSD of different orbiting satellite signals' pseudo random noise code (PRN) is illustrated in Fig. 1, where PRN 3 is a GEO satellite, PRN 8 is an IGSO satellite, and PRN 12 is a MEO satellite.

It can be observed in Fig. 1 that the PSDs of different satellites have the same envelope; however, the spectral line characteristics of different satellites are different, and

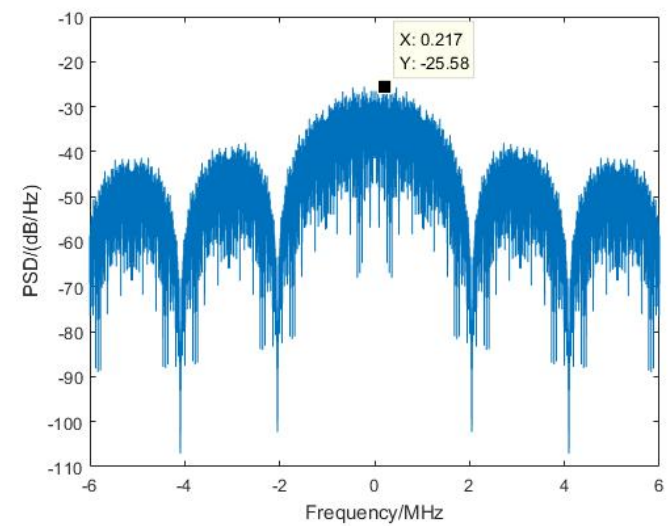

(a) The PSD of PRN 3

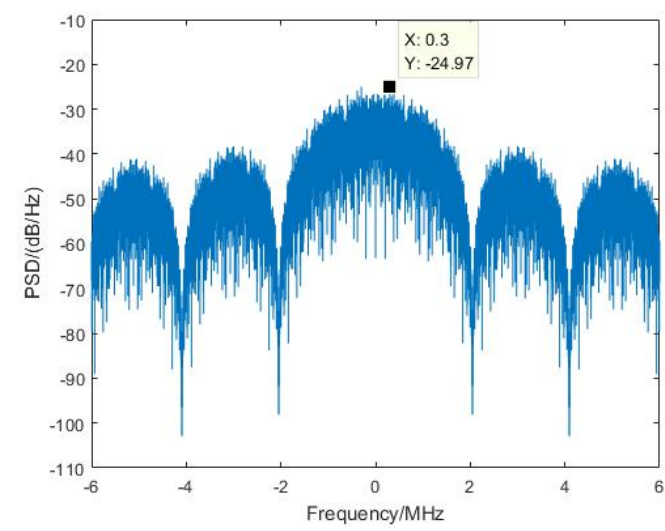

(b) The PSD of PRN 8

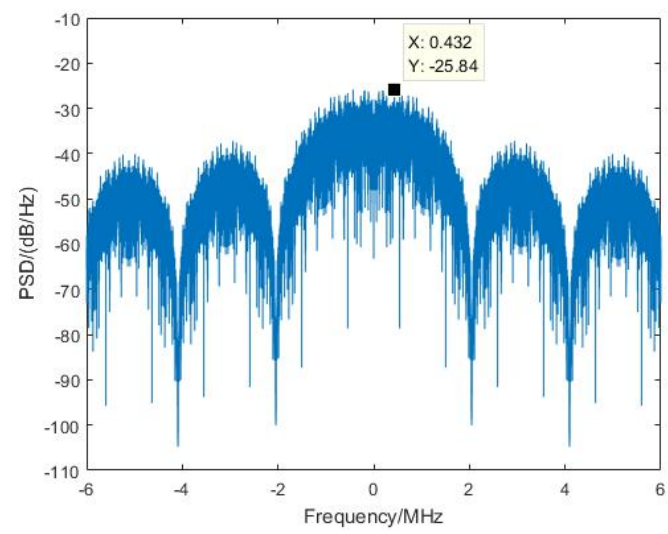

(c) The PSD of PRN 12

Fig. 1. The PSD of PRN 3, PRN 8 and PRN 12.

the maximum amplitude lines of each satellite are located at different frequency points.

\subsection{The EMS Criterion of the Satellite Tracking Missing}

The stability of the tracking loop in the navigation receiver depends on the measurement error of the loop. The sources of the error mainly include the square error of thermal noise $\sigma_{\mathrm{t}}$, phase jitter error $\sigma_{\mathrm{v}}$ and the square error of Alan $\sigma_{\mathrm{A}}$. The latter two errors are basically fixed values for the receiver, and $\sigma_{\mathrm{t}}$ is related to the $\mathrm{C} / \mathrm{N}_{0}$ [16]. The equation of $\sigma_{\mathrm{t}}$ is 


$$
\sigma_{\mathrm{t}}=\frac{180^{\circ}}{\pi} \sqrt{\frac{B_{\mathrm{L}}}{C / N_{0}}\left(1+\frac{1}{2 T_{\mathrm{d}}\left(C / N_{0}\right)}\right)}
$$

where $B_{\mathrm{L}}$ represents the noise bandwidth, and $T_{\mathrm{d}}$ denotes the integration duration time.

The conservative estimation method [17] for the loop tracking threshold is

$$
3 \sqrt{\sigma_{\mathrm{t}}^{2}+\sigma_{\mathrm{A}}^{2}+\sigma_{\mathrm{v}}^{2}}+\theta_{\mathrm{e}} \leq 45^{\circ}
$$

where $\theta_{\mathrm{e}}$ denotes dynamic stress error, whose value is generally the steady-state error value.

The combination of (2) and (3) reveals that there must be such a threshold of $C / N_{0}$ in the loop; the loop will lose the ability to track the satellite signal stably when the $C / N_{0}$ at the correlator output is less than this threshold. Therefore, the threshold of $C / N_{0}$ is used as the criterion for satellite tracking missing.

\subsection{The CWI Injection Experiment}

The block diagram and hardware setting of the CWI injection experiment are exhibited in Fig. 2. The experiment settings are presented as follows.

1) Navigation signal generator configuration: The satellite signals broadcast by the navigation signal generator are PRN 3, PRN 8, and PRN 12, and the signal power is the same.

2) Signal source setup: During the experiment, the adjustment range of the signal frequency is the Beidou B1I signal frequency, the adjustment step is $0.2 \mathrm{MHz}$, and the power adjustment step is $0.2 \mathrm{dBm}$. The signal generated by the signal source is attenuated and injected into the navigation receiver together with the navigation signal through the directional coupler.

3) Navigation receiver: The receiver used in the experiment comes from a certain type of unmanned aerial vehicle (UAV), which is also used in cars and helicopters. Its manufacturer is BDStar Navigation, and the internal board chip is adopted by many other companies. The navigation receiver supports Beidou B1I and B3I signals. Experiments are only conducted for the B1I signal frequency band considering that the laboratory only has B1I navigation signal simulators. Since the UAV is relatively weakly affected by multipath during the flight, the impact of multipath is ignored in the experiment. Its status data can be observed by the detection computer through a serial line.

4) The threshold of $C / N_{0}$ : As obtained in the repeated injection experiments, the electromagnetic interference threshold of the navigation receiver is $30 \mathrm{~dB} \cdot \mathrm{Hz}$. When the signal $C / N_{0}$ reaches this threshold, the satellite signal no longer participates in positioning. Different receivers have different $C / N_{0}$ thresholds according to the hardware design and software algorithms in the receiver.

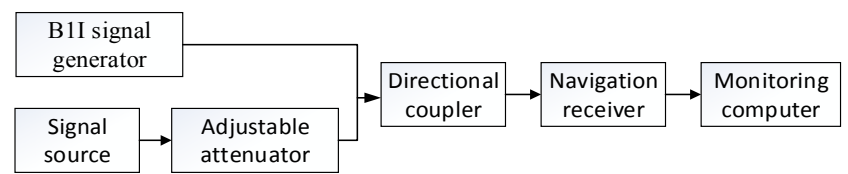

(a) The block diagram of CWI injection experiment

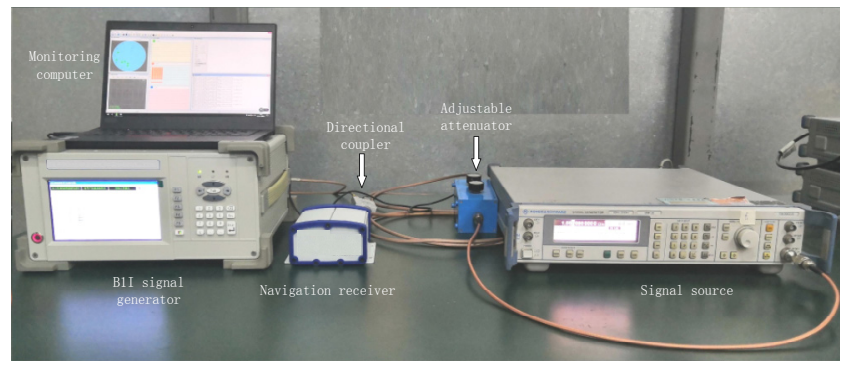

(b) The hardware setting of the CWI injection experiment

Fig. 2. The experiment of CWI injection.

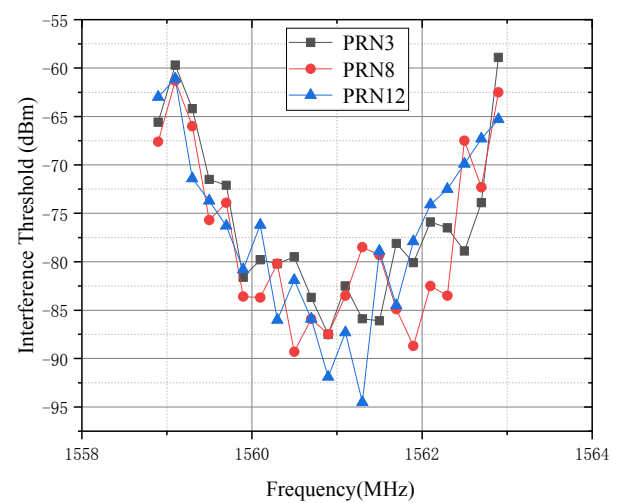

Fig. 3. The interference thresholds curve for PRN 3, PRN 8, and PRN 12.

The initial $C / N_{0}$ value received by the navigation receiver is a certain value. The interference threshold is measured when different satellite signals reach the $\mathrm{C} / \mathrm{N}_{0}$ threshold. The experimental results are presented in Fig. 3.

The following conclusions can be drawn from Fig. 3 .

1) Different satellites have different interference thresholds under the in-band CWI, and the lowest interference threshold occurs at different frequencies. This is related to the spectral line of the Beidou signal [18].

2) The bandwidth of the interference threshold curve is consistent with the bandwidth of the power spectrum of the navigation signal, as illustrated in Fig. 1.

\section{Investigation on the Prediction Model of B1I Navigation Receiver under In-band Dual-frequency CWI}

\subsection{The Prediction Model Derivation}

In this section, the prediction model of the satellite tracking missing in the Beidou navigation receiver is derived when the receiver faces dual-frequency CWI. The 
mathematical equation of the $C / N_{0}$ under single in-band CWI [19] is

$$
C / N_{0}=\frac{\left[\sqrt{2 P_{\mathrm{s}}} T_{\mathrm{d}} R_{0}(\tau) \operatorname{sinc}\left(\Delta f_{\mathrm{c}} T_{\mathrm{d}}\right)\right]^{2}}{L_{\mathrm{n}} N+\left[\sqrt{2 J} T_{\mathrm{d}} C_{\mathrm{n}} \operatorname{sinc}\left(\Delta f_{\mathrm{i}} T_{\mathrm{d}}\right)\right]^{2}}
$$

where $P_{\mathrm{s}}$ is the Beidou signal power, $N_{0}$ is the thermal noise power, $R_{0}(\tau)$ indicates the correlation between the received pseudo code and the locally copied pseudo code, $\Delta f_{\mathrm{c}}$ represents the difference between the signal frequency and the estimate of the carrier frequency, $J$ is the interference power, $C_{\mathrm{n}}$ denotes the spectral line coefficient related to interference frequency, $\Delta f_{\mathrm{i}}$ refers to the difference between the interference frequency and the estimate of the carrier frequency, and $L_{\mathrm{n}}$ is the processing gain applied to the noise.

It is assumed that there are two in-band CWI signals, with the frequency of $f_{1}$ and $f_{2}$, respectively. When the two CWI signals act alone, their interference thresholds are $J_{1}$ and $J_{2}$, respectively, the equation can be expressed as

$$
\left\{\begin{array}{l}
\left(C / N_{0}\right)_{\mathrm{m}}=\frac{\left[\sqrt{2 P_{\mathrm{s}}} T_{\mathrm{d}} R_{0}(\tau) \operatorname{sinc}\left(\Delta f_{\mathrm{c}} T_{\mathrm{d}}\right)\right]^{2}}{L_{\mathrm{n}} N+\left[\sqrt{2 J_{1}} T_{\mathrm{d}} C_{\mathrm{n} 1} \operatorname{sinc}\left(\Delta f_{\mathrm{i} 1} T_{\mathrm{d}}\right)\right]^{2}} \\
\left(C / N_{0}\right)_{\mathrm{m}}=\frac{\left[\sqrt{2 P_{\mathrm{s}}} T_{\mathrm{d}} R_{0}(\tau) \operatorname{sinc}\left(\Delta f_{\mathrm{c}} T_{\mathrm{d}}\right)\right]^{2}}{L_{\mathrm{n}} N+\left[\sqrt{2 J_{2}} T_{\mathrm{d}} C_{\mathrm{n} 2} \operatorname{sinc}\left(\Delta f_{\mathrm{i} 2} T_{\mathrm{d}}\right)\right]^{2}}
\end{array}\right.
$$

where $\left(C / N_{0}\right)_{\mathrm{m}}$ represents the threshold of the satellite tracking missing.

When the receiver is interfered with by the two CWI simultaneously, the calculation equation of the $\mathrm{C} / \mathrm{N}_{0}$ is

$$
\left(C / N_{0}\right)_{\mathrm{m}}=\frac{\left[\sqrt{2 P_{\mathrm{s}}} T_{\mathrm{d}} R_{0}(\tau) \operatorname{sinc}\left(\Delta f_{\mathrm{c}} T_{\mathrm{d}}\right)\right]^{2}}{L_{\mathrm{n}} N+J_{1 \mathrm{~g}} G_{1}+J_{2 \mathrm{~g}} G_{2}}
$$

where $G_{1}=2\left[T_{\mathrm{d}} C_{\mathrm{n} 1} \operatorname{sinc}\left(\Delta f_{\mathrm{ii}} T_{\mathrm{d}}\right)\right]^{2}, J_{1 \mathrm{~g}}$ and $J_{2 \mathrm{~g}}$ are the power of the two different frequency interference received by the receiver, and $G_{2}=2\left[T_{\mathrm{d}} C_{\mathrm{n} 2} \operatorname{sinc}\left(\Delta f_{\mathrm{i} 2} T_{\mathrm{d}}\right)\right]^{2}$.

According to (5) and (6), it can be obtained that

$$
\begin{aligned}
& J_{1 \mathrm{~g}} G_{1}+J_{2 \mathrm{~g}} G_{2}=J_{1} G_{1}=J_{2} G_{2} \\
& =\frac{\left[\sqrt{2 P_{\mathrm{s}}} T_{\mathrm{d}} R_{0}(\tau) \operatorname{sinc}\left(\Delta f_{\mathrm{c}} T_{\mathrm{d}}\right)\right]^{2}}{\left(C / N_{0}\right)_{\mathrm{m}}}-L_{\mathrm{n}} N .
\end{aligned}
$$

Then, it can be written as

$$
K=\frac{J_{1 \mathrm{~g}}}{J_{1}}+\frac{J_{2 \mathrm{~g}}}{J_{2}}=1
$$

where $K$ represents the suppression coefficient of in-band dual-frequency CWI. $K<1$ suggests that the current combination of dual-frequency interference power is still within the safety threshold and will not affect the stable tracking of a satellite signal in the receiver. $K \geq 1$ indicates that the current combination of dual-frequency interference power will cause the receiver to fail to track the satellite signal stably.

\subsection{The In-band Dual-frequency CWI Injection Experiment}

The configuration of the dual-frequency CWI experiment adds a set of signal sources to the configuration of the single-frequency CWI experiment. The configurations of the two sets of signal sources are the same. The experiment configuration is illustrated in Fig. 4.

During the experiment, two groups of in-band dualfrequency interference frequencies are selected for the experiment at will. In the first group, dual-frequency CWI frequencies are $f_{1}=1562.598 \mathrm{MHz}$ and $f_{2}=1559.598 \mathrm{MHz}$, and dual-frequency CWI frequencies in the second group

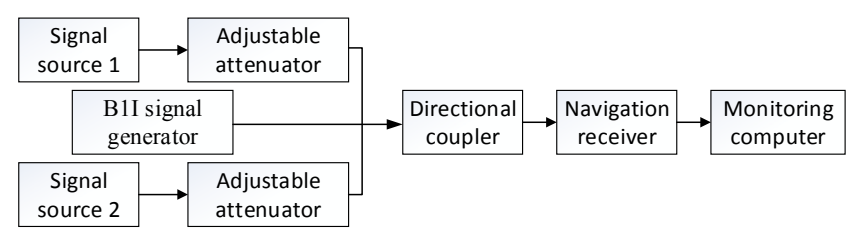

Fig. 4. The block diagram of the dual-frequency CWI injection experiment.

\begin{tabular}{|c|c|c|c|c|}
\hline & $f_{1}$ & $f_{2}$ & $K$ & Error/dB \\
\hline $\begin{array}{c}\text { Single } \\
\text { frequency } \\
\text { interference } \\
\text { threshold } \\
(\mathrm{dBm})\end{array}$ & -73.22 & -76.4 & - & - \\
\hline \multirow{4}{*}{$\begin{array}{c}\text { Dual } \\
\text { frequency } \\
\text { interference } \\
\text { threshold } \\
(\mathrm{dBm})\end{array}$} & -79.16 & -78.01 & 0.944923 & -0.49207 \\
\cline { 2 - 5 } & -74.02 & -78.94 & 0.974055 & -0.22833 \\
\cline { 2 - 5 } & -74.67 & -80.04 & 1.079656 & 0.665708 \\
\cline { 2 - 5 } & -74.48 & -83.05 & 0.964441 & -0.31449 \\
\hline
\end{tabular}

Tab. 1. The first group of dual-frequency CWI experiment.

\begin{tabular}{|c|c|c|c|c|}
\hline & $f_{3}$ & $f_{4}$ & $K$ & Error/dB \\
\hline $\begin{array}{c}\text { Single } \\
\text { frequency } \\
\text { interference } \\
\text { threshold } \\
(\mathrm{dBm})\end{array}$ & -86.09 & -75.66 & - & - \\
\hline & -91.94 & -76.9 & 1.011639 & 0.100511 \\
\cline { 2 - 5 } & -88.44 & -77.95 & 1.172304 & 1.380805 \\
\cline { 2 - 5 } & -87.84 & -79 & 1.131791 & 1.075325 \\
\cline { 2 - 5 } $\begin{array}{c}\text { Dual } \\
\text { frequency } \\
\text { interference } \\
\text { threshold } \\
(\mathrm{dBm})\end{array}$ & -87.58 & -79.97 & 1.080258 & 0.67055 \\
\cline { 2 - 5 } & -87.29 & -80.86 & 1.060573 & 0.510811 \\
\cline { 2 - 5 } & -86.72 & -81.92 & 1.10156 & 0.840163 \\
\hline
\end{tabular}

Tab. 2. The second group of dual-frequency CWI experiment. 


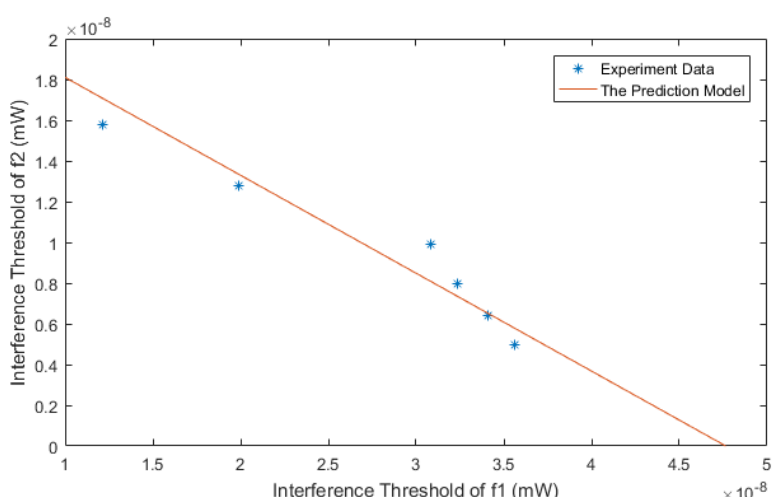

(a) The first group power combination of the dual-frequency CWI.

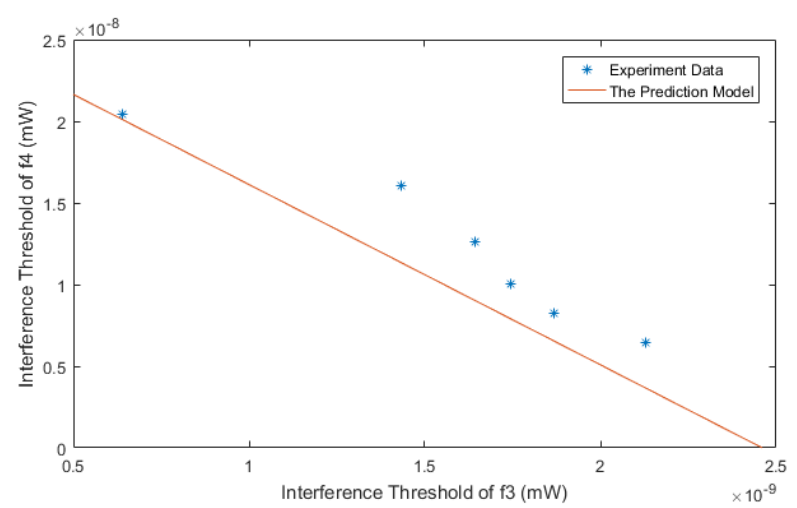

(b) The second group power combination of the dual-frequency CWI.

Fig. 5. The power combination of the dual-frequency CWI.

are $f_{3}=1562.098 \mathrm{MHz}$ and $f_{4}=1560.598 \mathrm{MHz}$. The experiment satellite is PRN 8 of the Beidou B1I signal. The twogroup experiment results are presented in Tab. 1 and Tab. 2. When the satellite is missed, the power combination of the dual-frequency CWI is demonstrated in Fig. 5. The error of the suppression coefficient is

$$
\text { Error }=20 \log K \text {. }
$$

The following conclusions can be drawn.

1) When the satellite is missed, the power combination between dual-frequency CWI is negatively correlated. As the power of one interference signal decreases, the other one increases.

2) The suppression coefficient $K$ of the prediction model under the combination of dual-frequency interference is basically around 1, and the error of the model is less than $2 \mathrm{~dB}$. The effectiveness of the dual-frequency interference model is verified by the experimental results.

The sources of experimental error mainly include the following aspects:

1) The error introduced by the experimental equipment. When the power of the CWI needs to be measured, it is necessary to disconnect the directional coupler from the navigation receiver and connect the directional coupler to the spectrum analyzer. The stability of the signal source output will bring a small error into the experiment results because the power measuring method is not real-time.
2) During the derivation of the $\mathrm{C} / \mathrm{N}_{0}$ formula under single-frequency interference, the quantization loss of ADC [20], [21] is not considered. However, the ADC quantization loss between dual-frequency CWI and singlefrequency CWI may be slightly different.

3) Errors in the measurement of interference threshold. To reduce this error, the interference threshold was measured three times during the experiment, and the average value was taken as the final result.

According to this model, it can be predicted whether the satellite tracking in the navigation receiver is lost under different dual-frequency interference combinations. Theoretically, this model can be extended to in-band multi-frequency interference combinations, however, due to the lack of verification conditions in the laboratory, the verification for multi-frequency interference combinations was not carried out.

\section{The Effect and Mechanism Analysis on Position Failure under Out-of- band CWI}

\subsection{The Experiment of the Out-of-band CWI for Navigation Receiver Positioning}

The experiment configuration is the same (Fig. 2). Before the experiment, a group of outdoor navigation signals was collected by the navigation receiver; then, the navigation signal generator sends the same navigation signal. Since the frequency range of the signal source is $10 \mathrm{MHz}-5 \mathrm{GHz}$, the sensitive frequency bands of the Beidou B1I navigation receiver are obtained. The experiment results are exhibited in Fig. 6.

As illustrated in Fig. 6, the navigation receiver has many sensitive frequency bands.

1) The center frequencies of the sensitive bands shown in Fig. 6(a)-(i) are 1/2, 1/3, 1/4, 1/5, 1/6, 1/7, 1/8, and $1 / 9$ of Beidou B1I signal frequency, respectively. This indicates that the interference whose frequency is located at the lower harmonic frequency of the navigation signal is the sensitive frequency of the receiver. The comparison reveals that when the lower the interference frequency, the narrower the corresponding sensitivity bandwidth, and the higher the minimum interference threshold.

2) In Fig. 6(j), the center frequency of the sensitive frequency band is around $41 \mathrm{MHz}$, the bandwidth is approximately $3.6 \mathrm{MHz}$, and the minimum interference threshold is $-42.6 \mathrm{dBm}$. In Fig. $6(\mathrm{k})$, the center frequency of the sensitive frequency band is around $3081 \mathrm{MHz}$, the bandwidth is approximately $4.2 \mathrm{MHz}$, and the minimum interference threshold is $-26.3 \mathrm{dBm}$. Notably, these two frequencies of navigation signal are not the low harmonic frequency of the B1I navigation signal (1561.098 MHz). 


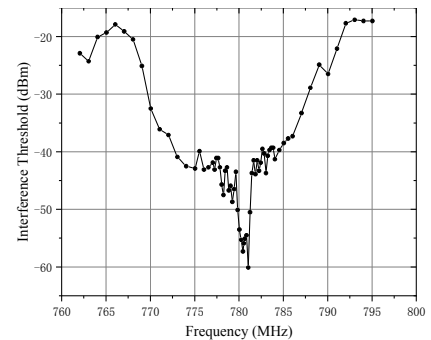

(a) $780.549 \mathrm{MHz}$

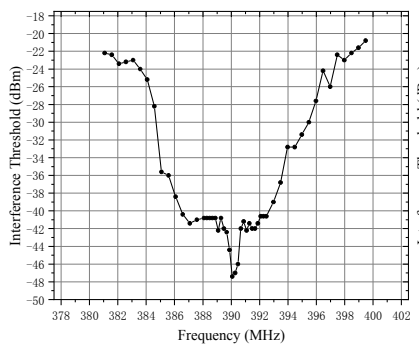

(c) $390.2745 \mathrm{MHz}$

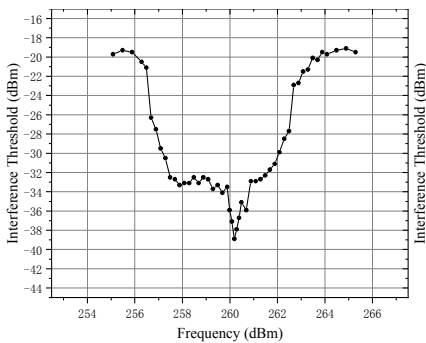

(e) $260.183 \mathrm{MHz}$

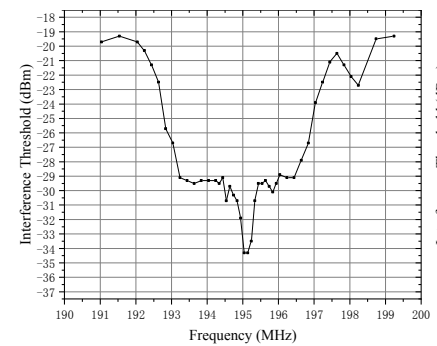

(g) $195.13725 \mathrm{MHz}$

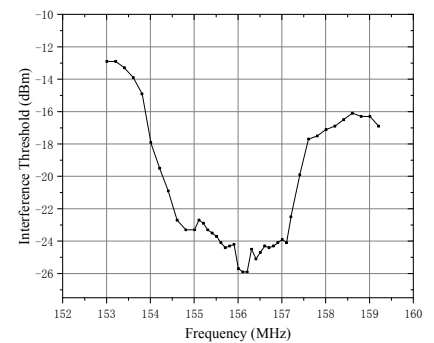

(i) $156.1098 \mathrm{MHz}$

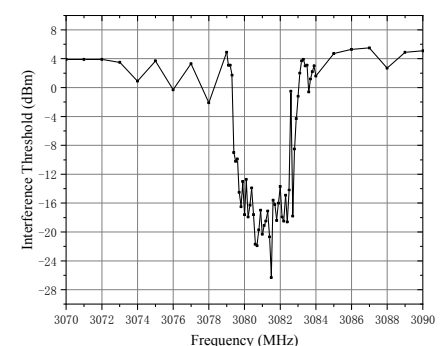

(k) $3081 \mathrm{MHz}$

Fig. 6. The sensitive frequency band of the navigation

\footnotetext{
receiver.
}

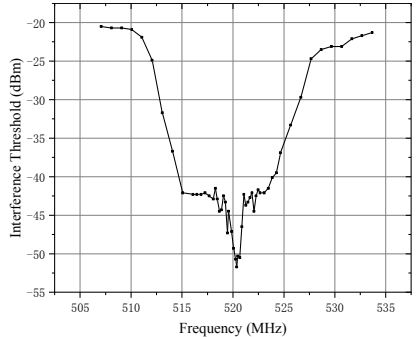

(b) $520.366 \mathrm{MHz}$

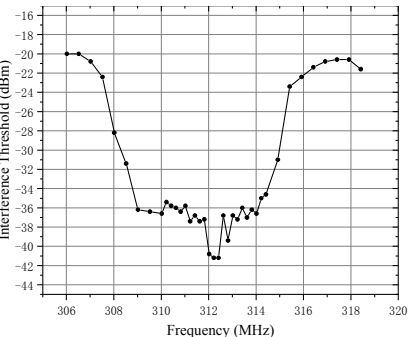

(d) $312.2196 \mathrm{MHz}$

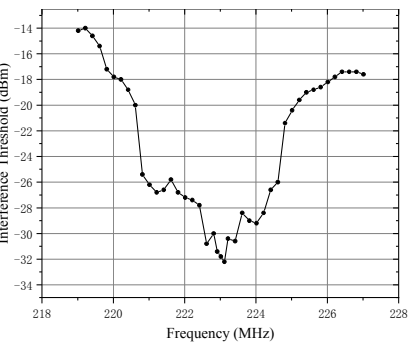

(f) $223.014 \mathrm{MHz}$

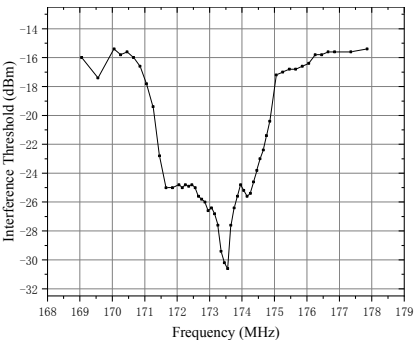

(h) $173.453 \mathrm{MHz}$

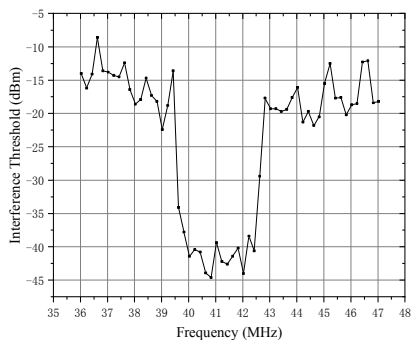

(j) $41 \mathrm{MHz}$

\subsection{The Mechanism of Out-of-band CWI}

As indicated in Fig. 6, the majority of the out-of-band sensitive frequency bands are the lower harmonic frequency bands of the B1I navigation signal. Based on this phenomenon, the mechanism has the following two possibilities:

1) The signal source produces high harmonic signals when it outputs an interference signal, which falls into the navigation signal frequency band. As a result, the navigation receiver positioning failure would occur.

2) The interference directly enters into the navigation receiver, and the lower interference power causes the navigation receiver positioning failure.

The following experiment is designed to verify the specific reason for the positioning failure in the navigation receiver. The experiment configuration is presented in Fig. 7. A band stop filter is added between the signal source and the directional coupler. The stopband of the band-stop filter is $1561.098 \pm 30 \mathrm{MHz}$, and the insertion loss of the band-stop filter is less than $1 \mathrm{~dB}$. Thus, it will prevent the higher harmonic frequencies of the interference from entering the receiver.

The interference signal frequency of the experiment is around $780.549 \mathrm{MHz}$. The experiment result is illustrated in Fig. 8.

After adding the band-stop filter, the interference threshold of the navigation receiver is basically unchanged, reflecting that the second possibility may be the main reason for the positioning failure in the navigation receiver.

The main nonlinear components in the navigation receiver contain a low noise amplifier (LNA) and a mixer [14]. When the input interference frequency of the LNA is the frequency of Fig. 6(a)-(i), the output signal frequency may contain the frequency of the navigation signal. This can explain the experimental phenomena in Fig. 6(a)-(i)

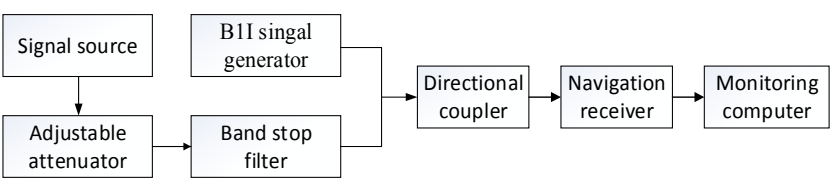

Fig. 7. The experiment with band stop filter.

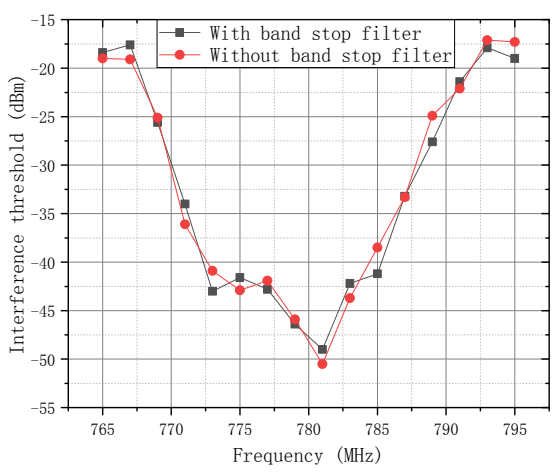

Fig. 8. The comparison of experiment results. 
instead of the experimental phenomena in Fig. 6(j)-(k) because $41 \mathrm{MHz}$ and $3081 \mathrm{MHz}$ are not the low harmonic frequency of B1I navigation signal. Therefore, the LNA is not the main reason for generating these sensitive frequency bands in Fig. 6 .

The mixer is an essential circuit in the navigation receiver. Because the frequency of the navigation signal is $1561.098 \mathrm{MHz}$, high-frequency signals are not easily sampled directly. Thus, a mixer is required to down-convert the received navigation signal. Hopefully, the output of the mixer only has the ideal useful signal frequency. However, there are many signals with other frequencies in the engineering.

The intermediate frequency (IF) generated by the mixer can be expressed as

$$
f_{0}=p f_{\mathrm{j}} \pm q f_{\mathrm{L}}
$$

where $f_{0}$ is the IF signal frequency, $f_{\mathrm{j}}$ is the interference frequency, and $f_{\mathrm{L}}$ is the local oscillator (LO) signal frequency [22].

ADS simulation software is a circuit simulation software developed by Agilent. Owing to its powerful functions in microwave circuit design, it is widely used in the design of receiver radio frequency circuits [23]. The circuit diagram of the mixer is established by ADS, as exhibited in Fig. 9.

PORT1 and PORT2 are the two input signal sources of the mixer. PORT1 represents the interference signal, and PORT2 represents the local oscillator (LO) signal of the mixer, whose frequency is $1520 \mathrm{MHz}$. The mixing circuit is composed of a directional coupler and a matching circuit. The low-pass filter circuit consists of capacitors and inductors, and the cut-off frequency of the low-pass filter is $100 \mathrm{MHz}$. Term 1 is the terminal load. A monitoring node is added to the positive terminal of the terminal load. Under the different input interference signal frequency, the output signal of the mixer can be used to qualitatively analyze the frequency component, as revealed by observing the monitoring node. In the simulation, the interference signal power is $-20 \mathrm{dBm}$, and the LO signal power is $10 \mathrm{dBm}$.

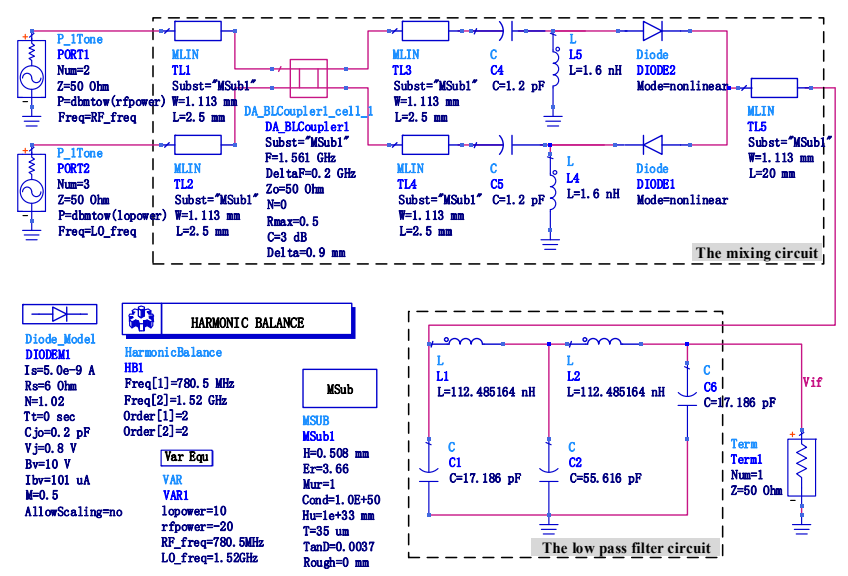

Fig. 9. The ADS simulation of the mixer.
Figure 10 exhibits the output signal of the mixer and filter when the interference signal frequencies are $41 \mathrm{MHz}$, $780.5 \mathrm{MHz}$, and $3081 \mathrm{MHz}$, respectively. The output signals contain a frequency of $41 \mathrm{MHz}$, which is the IF frequency in the navigation receiver. Once the interference signal is mixed into the IF, the navigation signal processing in the receiver will be affected, as well as the positioning performance of the receiver.

Considering that the detailed parameters of the mixer in the receiver are unknown, the ADS simulation results can only qualitatively analyze the mechanism of generating the above sensitive frequency bands.

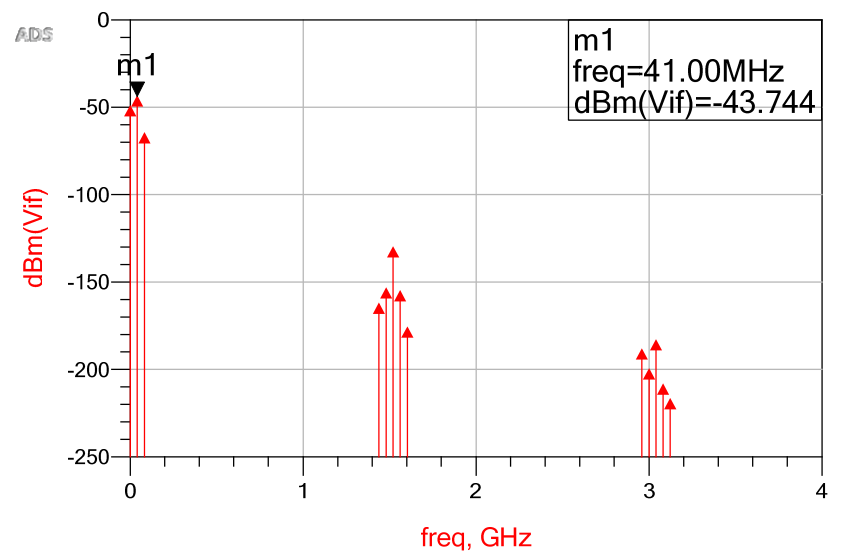

(a) The interference frequency is $41 \mathrm{MHz}$.

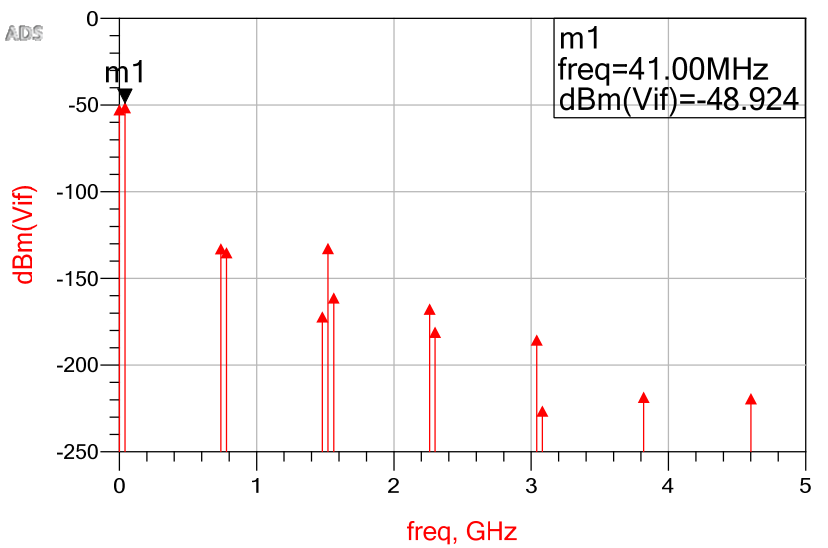

(b) The interference frequency is $780.5 \mathrm{MHz}$.

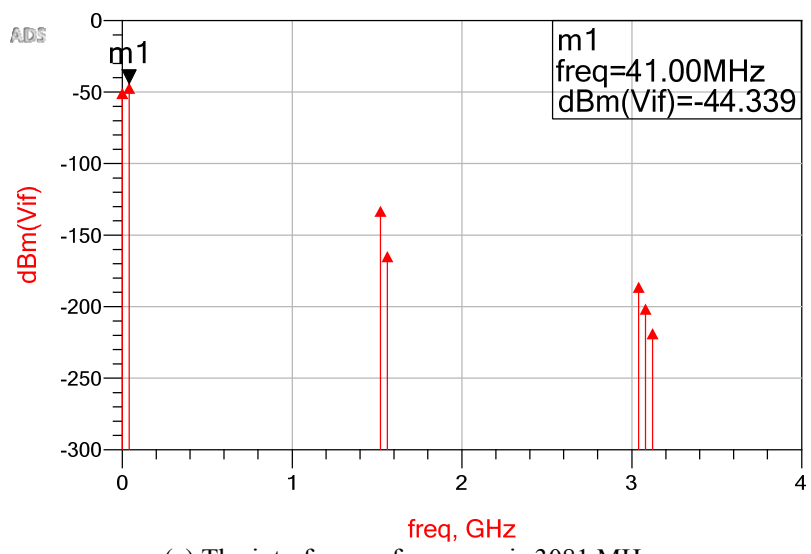

(c) The interference frequency is $3081 \mathrm{MHz}$.

Fig. 10. The output signal of the mixer and filter under the interference frequency. 
The analysis of the experiments and simulation demonstrates that the electromagnetic sensitive frequency bands of the navigation receiver are mainly concentrated in the low-order harmonic frequency band of the navigation signal and the IF signal frequency band. The main reason for this phenomenon is the nonlinearity of the mixer, that is, improving the selectivity of the RF front-end circuit contributes to suppressing the effects of out-of-band interference.

\section{Conclusion}

Through the CWI injection experiment on the navigation receiver, the interference threshold of the satellite track missing and the positioning failure are obtained, and the mechanism of the positioning failure of the navigation receiver is analyzed. The main conclusions are drawn as follows.

1) Different satellites have different interference threshold curves under in-band CWI, and the minimum interference threshold occurs at different frequencies due to the spectral line of the Beidou B1I signal.

2) When the satellite tracked in the navigation receiver is missed, the power combination of the dual-frequency CWI is negatively correlated under the dual-frequency CWI. Its relationship with the single-frequency interference threshold is expressed in (8). Experiment results indicate that the error of this model is less than $2 \mathrm{~dB}$.

3) The navigation receiver has many sensitive frequency bands. Regardless of the sensitive frequency band with the same frequency as the navigation signal, the other sensitive frequency bands are the secondary channel interference introduced by the mixer. Reducing this out-of-band interference can improve the frequency selectivity of the RF front-end.

\section{References}

[1] FAN, Y. Q., CHENG, E. W., WEI, M., et al. Effects of CWI of BDS receiver and analysis on the coupling path of electromagnetic energy. IEEE Access, 2019, vol. 7, p. 155885-155893. DOI: 10.1109/ACCESS.2019.2949462

[2] LI, W., CHENG, E. W., WEI, G. H., et al. Electromagnetic radiation effects forecasting method about in-band dual-frequency continuous wave for typical communication equipment. Systems Engineering and Electronics, 2017, vol. 38, no. 11, p. 2474-2480. DOI: 10.3969/j.issn.1001-506X.2016.11.04 (in Chinese)

[3] LI, B. Y., TANG, X. M., NIE, J. W., et al. Model analysis and design optimization for automatic gain control circuit in satellite navigation anti-jamming receivers. Journal of National University of Defense Technology, 2017, vol. 39, no. 6, p. 25-30. DOI: 10.11887/j.cn.201706005 (in Chinese)

[4] LIU, R. H., SHANG, P. Effect of different interferences on Beidou B1I signal receiver. Systems Engineering and Electronics, 2019, vol. 415 , no. 8 , p. 1705-1712. DOI: 10.3969/j.issn.1001506X.2019.08.05 (in Chinese)
[5] BAlAEI, A. T., DEMPSTER, A. G., LO PRESTI, L. Characterization of the effects of $\mathrm{CW}$ and pulse $\mathrm{CW}$ interference on the GPS signal quality. IEEE Transactions on Aerospace and Electronic Systems, 2009, vol. 45, no. 4, p. 1418-1431. DOI 10.1109/TAES.2009.5310308

[6] BETZ, J. W., KOLODZIEJSKI K. R. Generalized theory of code tracking with an early late discriminator. Part II: Noncoherent processing and numerical results. IEEE Transactions on Aerospace and Electronic Systems, 2009, vol. 45, no. 4, p. 1557-1564. DOI: 10.1109/TAES.2009.5310317

[7] LIU, Y. Q., RAN, Y. H., TING, K., et al. Code tracking performance analysis of GNSS signal in the presence of CW interference. Signal Processing, 2011, vol. 91, no. 4, p. 970-987. DOI: $10.1016 /$ j.sigpro.2019.09.022

[8] JANG, J., PAONNI, M., EISSFELLER, B. CW interference effects on tracking performance of GNSS receivers. IEEE Transactions on Aerospace and Electronic Systems, 2012, vol. 48 no. 1, p. 243-258. DOI: 10.1109/TAES.2012.6129633

[9] QU, B., WEI, J. L., TANG, Z. P., et al. Analysis of combined effects of multipath and $\mathrm{CW}$ interference on coherent delay lock loop. Wireless Personal Communications, 2014, vol. 77, no. 3, p. 2213-2233. DOI: 10.1007/s11277-014-1634-1

[10] QU, Z., YANG, Y., CHEN, J. Y. Continuous wave interference effects on ranging performance of spread spectrum receiver. Wireless Personal Communications, 2014, vol. 82, no. 1, p. 473-494. DOI: $10.1007 / \mathrm{s} 11277-014-2236-7$

[11] MANSSON, D., THOTTAPPILLIL, R., NILSSON, T., et al. Susceptibility of civilian GPS receivers to electromagnetic radiation. IEEE Transactions on Electromagnetic Compatibility, 2008, vol. 50, no. 2, p. 434-437. DOI: 10.1109/TEMC.2008.921015

[12] LIU, R. H., SHANG, P., LIANG, X. M. Conducted susceptibility test of Beidou airborne receiver. Electronic Measurement Technology, 2019, vol. 42, no. 3, p. 68-73. DOI: 10.19651/j.cnki.emt. 1802163

[13] WANG, J., WANG, Y., LIU, R. H., ZHANG, F. Z. Performance evaluation of Beidou system for airport single point service. Journal of Civil Aviation University of China, 2018, vol. 36, no. 5, p. 7-11.

[14] HegARTY, C. J., BOBYN, D., GRABOWSKI, J., et al. An overview of the effects of out-of-band interference on GNSS receivers. Navigation, 2020, vol. 67, p. 143-161. DOI: 10.1002/navi.345

[15] MENG, W. X., HAN, S., CHI, Y. G. Principles of Satellite Navigation and Positioning. Haerbin (China): Harbin Institute of Technology, 2013. ISBN: 9787560342214 (in Chinese)

[16] XIE, G. Principles of GPS and Receiver Design. Beijing (China): Publishing House of Electronics Industry, 2009. ISBN: 9787121090776 (in Chinese)

[17] KAPLAN, E. D., HEGARTY, C. J. Understanding GPS: Principles and Applications. 2nd ed. London (England): Artech House, 2005. ISBN: 1580538940

[18] ZHANG, T. Q., CHU, H. L. Continuous wave interference impact assessment tracking performance of Beidou civil B1I signal. In Proceedings of the 4th China Satellite Navigation Academic Annual Conference Electronic Collection. Nanjing (China), 2014 p. 47-52.

[19] BEK, M. K., SHAHEEN, E. M., ELGAMEL, S. A. Classification and mathematical expression of different interference signals on a GPS receiver. Navigation, 2015 , vol. 62 , no. 1 , p. $27-37$. DOI: 10.1002/navi.77

[20] AMOROSO, F. Adaptive A/D converter to suppress CWI in DSPN spread-spectrum communication. In MILCOM 1983 - IEEE 
Military Communications Conference. Washington (USA), 1983, vol. 3, p. 720-728. DOI: 10.1109/MILCOM.1983.4794795

[21] SAVASTA, S., MOTElla, B., DOVIS, F., et al. On the interference mitigation based on ADC parameters tuning. In Proceedings of the IEEE/ION Position Location and Navigations Symposium. Monterey (USA), 2008, p. 689-695. DOI: 10.1109/PLANS.2008.4570026

[22] CAO, X. W., LIU, N. A., CHEN, J., FU, W. H. Principle and Analysis of High Frequency Circuit. Xi'an (China): Xidian University Press, 2016. ISBN: 9787040447705 (in Chinese)

[23] LudWig, R., BOGDANOV, G. RF Circuit Design: Theory \& Applications. $2^{\text {nd }}$ ed., rev. London (UK): Pearson Education Inc., 2009. ISBN: 9780131471375

\section{About the Authors ...}

Qinglong ZHANG was born in 1987. He is a Ph.D. student at University of Shijiazhuang Campus of Army
Engineering University. His interest is in the electronic countermeasures of navigation system.

Yuming WANG was born in 1980. She received her Ph.D. degree at the Mechanical Engineering College. Her interest is in the electromagnetic compatibility.

Erwei CHENG was born in 1983. He is a Ph.D. student at Tsinghua University. His interest is in the simulation of environment effects of strong electromagnetic fields.

Liyun MA was born in 1984. She received her M.Sc. degree in 2011. Her interest is in the electromagnetic compatibility.

Yazhou CHEN (corresponding author) was born in 1975. He received his Ph.D. degree at the Mechanical Engineering College. His interests are in the simulation of environment effects of strong electromagnetic fields, electromagnetic environment effects and protection. 